\title{
Patent stereopsis with diplopia in random-dot stereograms
}

\author{
A. L. DUWAER \\ Department of Biological and Medical Physics, Erasmus University Rotterdam \\ Rotterdam, The Netherlands
}

\begin{abstract}
Fusional limits for an RD (random-dot) stereogram with overall horizontal retinal disparity due to the temporalward pulling of its two constituent RD patterns beyond the divergence limits of the eyes have been determined by using an afterimage method. Two criteria for fusion were used, viz, the perception of single local RD elements and the perception of stereoscopic depth in the "hidden" square of side $1.38 \mathrm{deg}$ with 8-min relative horizontal disparity. The diplopia thresholds were found to be in the range of 0.15-0.3 deg, and hence do not exceed the "classical" upper limit of about $0.3 \mathrm{deg}$, which has been reported for elementary line stereograms. The stereoscopic limits were found to be in the range of 0.5-1.3 deg, which is compatible with the precision of patent stereopsis from double images which has been reported for elementary line stereograms. The results of the present third look at the experiments performed by Fender and Julesz (1967) suggest that there are no special neuronal processes raising the fusional limits for RD stereograms above those for elementary line stereograms. Previous claims that such special neuronal processes may occur seem to be based on an evaluation of fusional limits obtained with different criteria for fusion. It is further argued that the major hysteresis effect that has been observed for fusional limits for RD stereograms should not be ascribed to a raising of the classical size of the diplopia threshold due to special neuronal processes initiated by RD stereograms, but to a lowering of the classical limits of patent stereopsis from double images due to the increased difficulty of solving the correspondence problem in RD stereograms.
\end{abstract}

In a classical paper, Ogle (1952) described the various percepts that occur when the horizontal disparity between the half-images on the retinas of a simple line stimulus is increased. First the amount of stereoscopic depth relative to the fixation target (without horizontal disparity) increases. Then diplopia occurs while depth can still be perceived. The disparity at which diplopia begins is usually called the diplopia threshold, while the disparity region determined by the diplopia thresholds at positive and negative disparities is known under the name of Panum's area. The "classical" upper limit of the diplopia threshold for horizontal disparity in elementary line stimuli under normal viewing conditions (without stabilizing the images on the retinas), including conditions with slow disparity increase, amounts to about $0.3 \mathrm{deg}$ in the fovea (Duwaer \& Van den Brink, 1981a). ${ }^{1}$ When the disparity is increased beyond the diplopia threshold, the relative depth increases further until it deteriorates strongly at a disparity level that is usually far above the diplopia threshold. The validity of stereoscopic depth perception from double images has been demon-

I would like to thank G. van den Brink, Ch. M. M. de Weert, and A. E. H. Peters for helpful discussions, and the Netherlands Organization for the Advancement of Pure Research for the funds which made this investigation possible. My mailing address is: Philips Research Laboratories, WO-p, P.O. Box 80000, 5600 JA Eindhoven, The Netherlands. strated by, for example, Blakemore (1970), Ogle (1952, 1953, 1955), Reading (1970), and Westheimer and Tanzman (1956).

In 1967, Fender and Julesz published a paper which repudiated Ogle's classical paper in contemporary theorizing about stereopsis and binocular fusion. Fender and Julesz claim that slowly increasing the overall (uncrossed) horizontal disparity on the retinas between the half-images (which were stabilized on the retinas) of a foveally presented random-dot (RD) stereogram (consisting of a surround and a "hidden" square with a relative crossed horizontal disparity of $8 \mathrm{~min}$ ) causes the "fused" percept of a single RD pattern with a square floating in front of the surround to be disrupted and diplopia to occur at a disparity (amounting to $2 \mathrm{deg}$ ) that is an order of magnitude larger than the diplopia thresholds reported thus far for elementary line stimuli. Fender and Julesz, and many others (e.g., Nelson, 1975), interpret this effect as entailing a major extension of Panum's fusional area by special neuronal processes that are activated only when the horizontal disparity in a complex stimulus is slowly increased.

However, an alternative interpretation to be tested in this paper is that these large fusional limits are not the enlarged disparity limits at which diplopia starts, but indicators of the much higher disparity values at which the perception of stereoscopic rel- 
ative depth disappears. This would imply that it was not necessary to assume that special neuronal processes might raise the diplopia thresholds in complex stimuli, and that the occurrence of diplopia was overlooked by previous investigators. The latter would not be surprising, since diplopia tends to be masked in RD patterns. A diplopic RD pattern is, at first sight, just a plain RD pattern with an inconspicuously different internal configuration in which binocular rivalry appears to be stabilized, perhaps because of the stereoscopic depth (cf. Ogle \& Wakefield, 1967). Moreover, Fender and Julesz (1967) explicitly state that the individual dots of their RD stereogram were just at or below the limit of resolution under their experimental conditions, which involved binocular stabilization, while Diner (1978, p. 90) reports that Fender and Julesz did not pay much attention to binocular rivalry, and Crone and Hardjowijoto (1979) - who confirmed Fender and Julesz's findings for a nonstabilized RD stereogram, of which the angular dimensions were a factor of 10 larger-state that their subjects were instructed to avoid conscious recognition of individual dots in the RD stereogram by continuously varying direction of gaze. Now, continuous variation of direction of gaze is known to increase the stability of the percept of stereoscopic depth from double images in elementary line stimuli (Linschoten, 1956; Ogle, 1952; Reading, 1970).

The above alternative interpretation of the large fusional limits for RD stereograms would imply that it should be possible to perceive stereoscopic depth in these stereograms while the individual dots are perceived in diplopia due to overall horizontal retinal disparity. A priori, this would not be surprising, since Marlowe (1969, referred to in Julesz, 1971) has shown that stereoscopic depth can be perceived in random-line stereograms, even though, due to cyclodisparities of up to at least $15 \mathrm{deg}$, the lines are seen in diplopia.

We tested the above alternative interpretation of the results obtained by Fender and Julesz (1967) by determining fusional limits for the RD stereogram used by them with two criteria for fusion: the perception of single (local) elements (in particular the elements at which the subject directs his/her gaze) and the perception of stereoscopic depth in the "hidden" figure. Our alternative interpretation would have to be rejected if the fusional limits corresponding to the singleness criterion were much larger than the classical upper limit of $0.3 \mathrm{deg}$, if the fusional limits obtained with the stereoscopic depth criterion were not predictable from the data on the precision of stereoscopic depth perception from double images of line stimuli reported by Blakemore (1970), Ogle (1953), and Reading (1970), and/or if the fusional limits obtained with the two criteria for fusion did not differ.
In the present study, overall uncrossed horizontal disparity was not induced on the retinas via binocular image stabilization (as done by Fender \& Julesz, 1967), but by pulling the images temporalward beyond the divergence limits of the eyes (as suggested by Fender \& Julesz, 1967). The amount of retinal disparity at the fusional limit was determined by a straightforward afterimage method, which has the three major advantages that it is very easy to apply, that it requires no attachments to the eyes, and that it requires no additional visual stimuli or tasks during the measurements of fusional limits that might interfere with visual performance, such as the use of dichoptic nonius lines and the need to assess their relative positions in the subjectivenonius-line method (Duwaer, 1982a, 1982b). By not stabilizing the images on the retinas, we avoided the complications associated with this type of stimulation, most notably the occurrence of fading of perceived images (Diner, 1978; Ditchburn, 1973).

For the sake of clarity, we wish to emphasize that there is a major difference between (1) the present experiment and Fender and Julesz's (1967), and (2) recent studies on the maximum disparity that can elicit depth perception (e.g., Richards, 1977; Tyler and Julesz, 1980). In the latter studies, the amount of horizontal disparity in the hidden figure relative to its surround is varied. This provides data on the ability to discern a depth difference between the hidden figure with a large horizontal disparity and the surround without horizontal disparity. The former studies vary the amount of overall horizontal disparity in both hidden figure and surround with a fixed relative disparity, amounting to $8 \mathrm{~min}$, between hidden figure and surround. This provides data on the ability to resolve this disparity difference of $8 \mathrm{~min}$ on the basis of a depth difference between hidden figure and surround when both are presented at large horizontal disparities. Both types of measurements require that stereoscopic depth can be elicited at large horizontal disparities. However, the measurements with varying overall disparity and fixed relative disparity require in addition that the amount of this stereoscopic depth varies when the disparity varies.

\section{METHOD}

\section{Stimulus}

The fusional limits were determined for a binocular pair of random-dot patterns, as described by Julesz (1971). The entire pattern (see Figure 1) subtended $3.43 \mathrm{deg}$ in the visual field. It consisted of a $100 \times 100$ array of square-shaped picture elements subtending a little over 2 min each, together with a "hidden" square of side $83 \mathrm{~min}$ with a crossed relative horizontal disparity of $8 \mathrm{~min}$. When the stereogram was fused, the hidden square was seen in front of the surround. The patterns were presented as copies on transparent film in front of a practically homogeneously illuminated background. Each pattern was attached to a beam splitter, which combined the pattern optically 

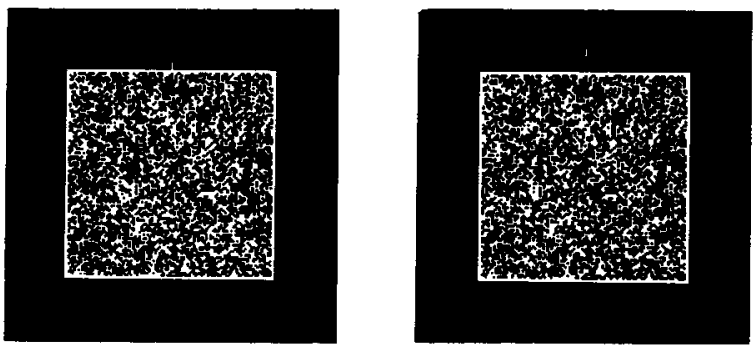

Figure 1. The RD stereogram used in the present study.

with a black mask containing a circular hole, of diameter $6 \mathrm{~min}$, which was given the same perceived location as the 6-min square (defined on three sides by a white border) in the upper righthand corner of the hidden square in the stereogram. An afterimage at the position of the above 6-min square, which will be called the "fixation square" from now on, could be imprinted on the retinas with the aid of a photographic flash (duration $10 \mathrm{msec}, 6 \mathrm{log}$ units above perception threshold) behind the circular hole. Each pattern with beam splitter and masked flashlight was positioned on a holder (see Figure 2). The two holders could be moved simultaneously equal distances in opposite directions by rotating a knob at the end of a rod with a counterclockwise screw-thread through one holder on one half and a clockwise screw-thread through the other holder on the other half. The patterns were presented at a distance of $50 \mathrm{~cm}$ from the subject's eyes. Their "initial separation" was that at which the patterns could be fused with parallel visual axes. The patterns were also presented at optical infinity with the aid of appropriate lenses in front of the subject's eyes. The separation between the patterns was always larger than or equal to the initial separation defined above. There was no noticeable blur due to the accommodation-convergence coupling and no noticeable change in apparent size. The mean luminance of the perceived patterns amounted to $33 \mathrm{~cd} / \mathrm{m}^{2}$.

\section{Procedure}

The subject was positioned in the experimental apparatus and his or her head was immobilized with a bite bar. The subject was then given the optical correction necessary for optimum visual acuity, and instructed to try to fuse the two monocular patterns with the eye muscles relaxed. When "he" correctly reported seeing a square in front of the surround, indicating that fusion had been achieved, he was instructed to shorten the

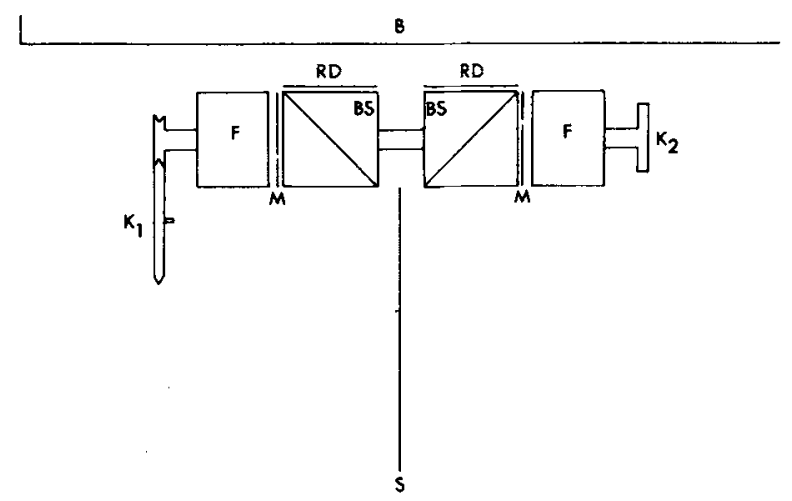

Figure 2. Experimental apparatus. $\mathbf{F}=$ flashlight, $\mathbf{R D}=\mathbf{R D}$ pattern, $M=$ mask with hole, $K_{1}$ and $K_{2}=$ knobs to adjust the lateral separation between the two RD patterns, $B S=$ beam splitter, $B=$ background field, $\mathrm{S}$ = separation screen. time necessary for fusion to a few seconds by repeatedly disrupting the fusion by closing the eyes or by looking away from the RD stereogram. He then slowly increased the separation between the RD patterns until two patterns were seen. The separation was then decreased to the initial value (at which the visual axes of the two eyes have to be parallel for fusion). When fusion had been reestablished and the two vertical dichoptic nonius lines above the stereogram-which were physically aligned with the surround of the hidden square in the stereogram-were perceptually aligned to within about $5 \mathrm{~min}$, the separation between the patterns was increased again. This sequence was repeated for at least half an hour, until the subject was familiar with the perceptual changes in the appearance of the fixation square and in the stereoscopic depth due to temporalward pulling of the patterns. All subjects stated that the perception of depth disappeared when the separation became too large. Except for two subjects, who were found to suppress the image in one of the eyes, they also stated that this disappearance of depth was preceded by doubling of the fixation square. The subject was then informed of his task, namely to collect data on the onset of doubling of the fixation square and the onset of a percept without depth, and was finally familiarized with the actual measuring procedure.

Each determination of a fusional limit started with adjustment of the separation between the RD patterns to their initial value. When the hidden square was seen with the appropriate stereoscopic depth, and the two dichoptic nonius lines were aligned to within about $5 \mathrm{~min}$, the subject increased the separation between the patterns until the mean relative position of the nonius lines started to change as a result of the pulling. This indicated that the separation was no longer fully compensated for by divergent vergence eye movements, so that retinal disparity started to arise. The subject then switched his gaze to the fixation square, that is, to a fixation position similar to that used by Fender and Julesz (1967). The separation was then slowly increased (at a rate of about $2 \mathrm{~min} / \mathrm{sec}$ ) until the criterion for loss of fusion (doubling of fixation square or no stereoscopic depth) was reached. It should be remembered that the fixation square is located at the upper right corner of the hidden square so that both criteria are likely to be based on percepts primarily in the fovea. The subject then looked at a portion of the RD pattern $15 \mathrm{~min}$ below the fixation square and actuated the two photographic flashes by pressing a button, thus imprinting an afterimage of the location of the fixation square in each eye (see Figure 3A). It should be noticed that this fixation square stimulates disparate retinal locations in the two eyes, since it is displaced to the left for the left eye and to the right for the right eye with respect to the corresponding points in the foveolas ( $f_{l}$ and $f_{r}$, see Figure 3A) of the two eyes. The subject then quickly reduced the separation between the $R D$ patterns to the initial value and waited at least $10 \mathrm{sec}$ until fusion was regained, the nonius lines had approximately their initial relative position, and the pair of afterimages had faded to invisibility. The subject then looked at the fixation square and actuated the two photographic flashes, giving, in both eyes, a second afterimage at the position of the fixation square (see Figure 3B). This second pair of afterimages will approximately mark the corresponding points in the foveolas ( $f_{1}$ and $f_{r}$, see Figure $3 B$ ) of the two eyes. In order to regenerate the first pair of afterimages and to increase the time during which the afterimages were visible (Magnussen \& Tjorjussen, 1974), the subject then looked at a TV screen (dimensions, $7.4 \times 4.9 \mathrm{deg}$; mean luminance level, $19 \mathrm{~cd} / \mathrm{m}^{2}$; fixation distance, $200 \mathrm{~cm}$ ), the luminance of which was sinusoidally modulated with a frequency of $2.7 \mathrm{~Hz}$ and a depth of $100 \%$. In order to obtain a measure for the disparity of the fixation square during imprinting of the first pair of afterimages, the subject was asked to estimate the horizontal displacement $(x)$ of the center of the first afterimage (the upper and initially dimmer one) with respect to the center of the second afterimage, with reference to a scale provided by horizontal and vertical scale units of $15 \mathrm{~min}$ depicted on the TV 


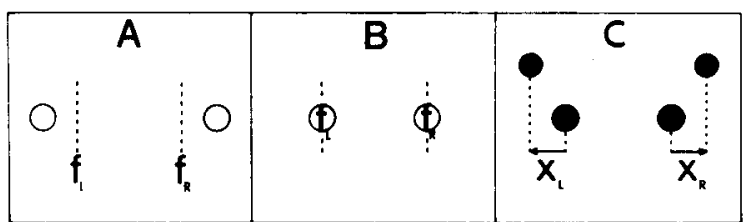

Figure 3. Principles involved in the afterimage methods. The visual axis of the left and right eye (indicated by $f_{1}$ and $f_{r}$, respectively) and the positions of the fixation square (indicated by open circles) are schematically represented for the conditions that the RD patterns are pulled temporalward (A) and that the RD patterns are at their initial separation (B). The solid dots in $C$ represent the afterimages imprinted under condition $A$ (upper dots) and condition $B$ (lower dots). The coordinates $x_{1}$ and $x_{1}$ are estimated by the subject. The discrepancy $\left(x_{r}-x_{1}\right)$ is a measure for the horizontal disparity induced by the temporalward pulling of the RD patterns.

screen (see Figure 3C). The horizontal displacement $(x)$ is defined to be positive when the first afterimage is displaced to the right with respect to the second afterimage, and negative when the first afterimage is displaced to the left. The estimation of $x$ was done for the left eye by closing the right one (giving $x_{1}$ ) and for the right eye by closing the left one (giving $x_{T}$ ). The subject plotted, on graph paper, the coordinates thus observed $\left(x_{1}\right.$ and $\left.x_{T}\right)$. He was advised to increase the accuracy of the estimation as much as possible by using all the available time (about $1 \mathrm{~min}$ ) and by looking at the afterimages alternately with the left and right eyes. It was mentioned that it was quite normal to find different coordinates for the two eyes, or in successive trials, and that the subject's only concern should be an accurate and objective estimation of what he actually perceived. The diameter of the afterimages amounted to about 0.4 scale units ( $6 \mathrm{~min}$ of $\mathrm{arc}$ ) during the estimation of the coordinates.

When the afterimages had finally faded to invisibility against the temporally modulated background provided by the TV screen, the next trial was started. Trials with the singleness/doubleness criterion, trials with the depth criterion, and trials in which the subject did not increase the separation between the RD patterns at all were alternated until 12 pairs of coordinates were obtained for each of the three conditions.

In a subsequent series of experiments, the accuracy and validity of the estimates of the coordinates of the afterimages were checked under the same experimental conditions by imprinting afterimages with known physical coordinates and using masks with two holes for each eye. The coordinates were chosen so as to mimic the coordinates estimated during the actual measurements.

To perform the experiments, it took the subject two sessions of about $4 \mathrm{~h}$ each with several rest periods included in each session. The two sessions were performed on different days.

\section{Data Analysis}

Since the afterimages provide retinal landmarks showing the direction of the visual axis during imprinting (Ditchburn, 1973), the discrepancy $\left(x_{r}-x_{1}\right)$ between the coordinates in the two eyes is a measure of the retinal disparity introduced by the temporalward pulling of the RD patterns. Note that $\left(x_{r}-x_{1}\right)=\left|x_{r}\right|+\left|x_{1}\right|$ in Figure 3 .

It should be noted that, because of the possible occurrence of a nonzero fixation disparity during imprinting of the afterimages at the initial separation (see the subsection entitled "Mean horizontal fixation disparity during fusion of unpulled RD stereogram" in Results and Discussion below), this induced retinal disparity, as measured by our afterimage method, may differ from the actual retinal disparity during observation of temporalward pulled RD patterns. However, the perceived relative positions of the two dichoptic nonius lines above the stereogram indicated that this fixation disparity was less than 0.1 deg in all subjects tested.
Means and standard deviations of the discrepancies $\left(\mathrm{x}_{\mathrm{T}}-\mathrm{x}_{1}\right)$ were determined in scale units and converted to minutes of arc using the results of the calibration measurements which indicated that the functional relation between the estimated discrepancy $\left(d_{e}=x_{T}-x_{1}\right)$ between the coordinates in the two eyes and the physical discrepancy $\left(\mathrm{d}_{\mathrm{ph}}\right)$ could be represented by the relation $\mathrm{d}_{\mathrm{e}}=\mathrm{d}_{\mathrm{ph}} / \alpha$, in which the calibration factor $\alpha$ was found to vary between 0.76 and 0.94 (apparently independent of the optical corrections applied), revealing systematic overestimation of the coordinates (cf. Duwaer, 1982a). This overestimation was corrected for each subject separately with the aid of the relation: 1 scale unit $=\alpha \times 15 \mathrm{~min}$ of arc ( $\alpha$ being determined individually for each subject).

\section{Subjects}

The experiments were started with 10 subjects, aged 19 to 40 years. One of the subjects was rejected since he was unable to perceive stereoscopic depth in the RD stereogram. The data obtained from a second subject were discarded when the calibration measurements showed that she had misunderstood the afterimage method. The present study is thus based on data obtained from 8 subjects ( 7 males and 1 female, J.S.), all of whom had normal stereopsis (better than $1 \mathrm{~min}$, according to the TNO random-dot test) and normal monocular Landolt-C visual acuities (better than 1). Subjects A.L.D., P.B., and R.S. were corrected for myopia (with $-0.75,-2.25$, and $-2.0 \mathrm{D}$, respectively), and Subjects R.R. and J.S. for hypermetropia (with +2.0 D and +0.75 D, respectively). Subjects C.J.D., B.W., and B.F. were emmetropic. Various tests in which the eyes were covered alternately with and without temporalward pulled images showed conclusively that two subjects (B.W. and B.F.) exhibited a "suppression effect"; that is, they tended to suppress the image in one of the eyes as soon as corresponding retinal locations received stimuli with dissimilar contours-they saw the image in only one eye, with the other eye merely contributing to the percept by generating stereoscopic depth. This is a known perceptual phenomenon. It may also be observed when subjects without this suppression effect look at an RD stereogram with one of the monocular patterns blurred (see, e.g., Figure 3.10-3 in Julesz, 1971).

Subject C.J.K. had an eccentric fixation point $(0.5 \mathrm{deg}$ nasalward in the visual field) for the right eye. This was discovered by getting the subject to look through a mask with a small, 1 -mm-diam hole rotating in a 3.5 -mm-diam circle place about $1.5 \mathrm{~cm}$ in front of his eye (cf. Drysdale, 1975), thereby visualizing, for this subject, both the fixation target in a luminous surround and his retinal blood vessels.

\section{RESULTS AND DISCUSSION}

Raw data obtained from one of the subjects (R.R.) are presented in Figure 4A (assessment of fusional limits) and Figure 4B (assessment of calibration factor). The calibration factor, $\alpha$, was determined by fitting the relation "estimated disparity" $=1 / \alpha \times$ "physical disparity" to the experimental points and the mirror images of these points in the third quadrant (the points $-x,-y$ ) by linear regression; this strategem ensures that the regression line passes through the origin. The longer broken line in Figure $4 C$ indicates this regression line. For Subjects R.R. and R.S., a separate calibration factor was calculated and used for disparities smaller than 3 scale units, since the data points indicated a systematic larger overestimation in this range. The corresponding regression line is indicated in Figure 4B by the shorter broken line. 

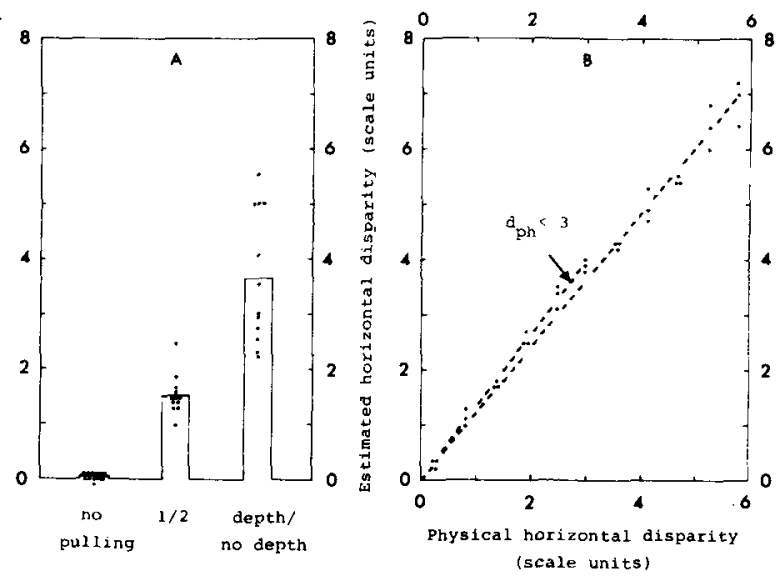

Figure 4. Raw data from determination of fusional limits (A) and of calibration factor (B). Each dot represents the horizontal disparity estimated from one pair of afterimages. The heights of the bars in $A$ represent mean values of the estimated horizontal disparity. The broken lines determine the calibration factor $\alpha$ (see text). The data were obtained from Subject R.R.

The calibration factors thus obtained amounted to 0.94 for Subject A.L.D., 0.83 for C.J.K., 0.94 for P.B., 0.82 and $0.76(<3$ scale units) for R.R., 0.92 and 0.79 ( $<3$ scale units) for R.S., 0.93 for B.W., and 0.85 for B.F. The data, converted to minutes of arc for all eight subjects, are presented

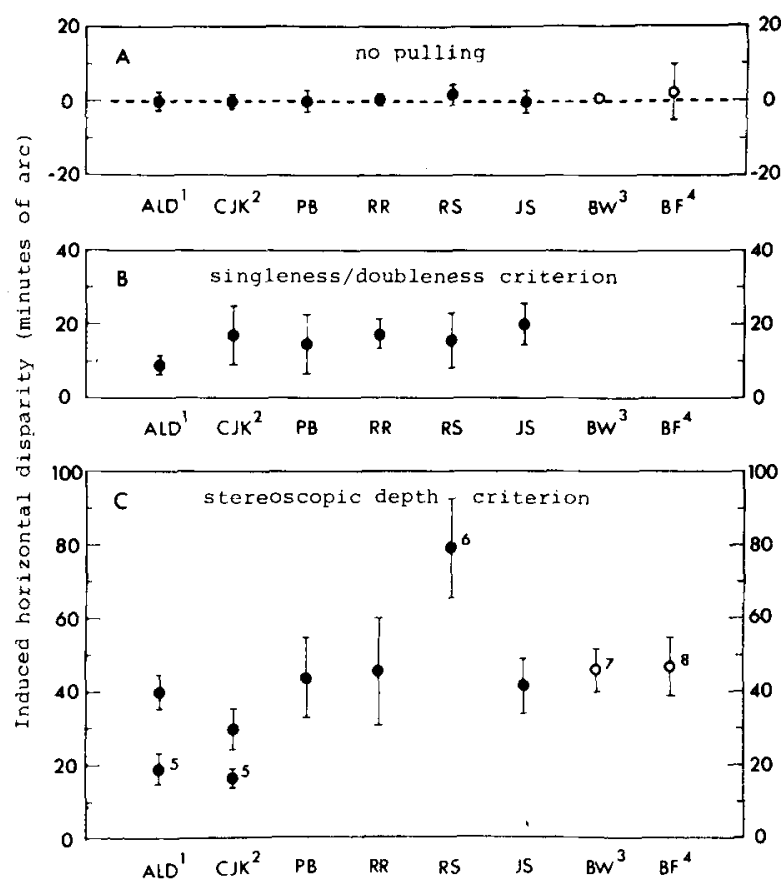

- Figure 5. Mean (circles) and standard deviations (SDs) (length of vertical bars through circles $=2$ SDs) of the induced horizontal disparities as estimated from 12 pairs of afterimages obtained without pulling (A), with temporalward pulling and the singleness/doubleness criterion (B), and with temporalward pulling and the stereoscopic depth criterion $(C)$. The data are plotted in minutes of arc for the eight different subjects (indicated by their initials). Various special features are indicated by raised numbers, which are explained in the text. in Figure 5: Figure 5B shows the data obtained using the singleness/doubleness criterion, and Figure 5C shows those obtained using the stereoscopic depth criterion. Figure 5A depicts the data obtained when the RD patterns were not pulled at all. The mean induced horizontal disparity should then be zero; this was actually found to be the case within the experimental accuracy. Various characteristics of the test subjects or experimental runs are indicated by raised numbers as follows: (1) well-practiced subject (the author), (2) practiced subject with eccentric right-eye fixation and anomalous correspondence, (3) subject with a tendency to suppress the image in the left eye, (4) subject with a tendency to suppress the image in the right eye, (5) stereoscopic limit obtained with rigid fixation of gaze, (6) fusional limit obtained using disappearance of all cues pointing to the "hidden" square as criterion for disruption of fusion, (7) simultaneous disruption of stereoscopic depth and suppression of image in left eye at outer boundary of RD pattern, and (8) suppression of image in left eye disrupted at outer boundary of $R D$ pattern at $58.1 \pm 8.5 \mathrm{~min}$.

Various aspects of the data will now be discussed in detail.

\section{Diplopia Thresholds for the RD Stereogram}

The onset of diplopia in subjects with normal binocular single vision without a suppression effect was found to occur at mean induced horizontal disparities of 9-20 min. This indicates that the diplopia thresholds certainly do not exceed classical limits reported for simple line stimuli (Duwaer \& Van den Brink, 1981a). It is interesting to note that the diplopia thresholds for the RD stereogram do not exceed the diplopia thresholds for even a binocularly stabilized bar (amounting to $0.4 \mathrm{deg}$ ), as reported by Diner (1978).

Although the possibility of depth and diplopia coexisting in RD stereograms was explicitly denied on theoretical grounds by Nelson (1975), those of our subjects without a suppression effect reported that they could see stereoscopic depth at and beyond the onset of diplopia.

The two subjects with the tendency to suppress the image in one of the eyes were found to see doubling of the image at the left and right outer boundaries of the RD stereogram for mean induced horizontal disparities larger than 47-58 min.

\section{Stereoscopic Limits for the RD Stereogram}

Stereoscopic depth was found to disappear at mean induced overall horizontal disparities that were "typically" 29-46 min with extreme values of 16-19 and $80 \mathrm{~min}$.

The extreme value of $80 \mathrm{~min}$ was obtained from a subject who found it difficult to determine when stereoscopic depth disappeared because the hidden square remained visible even when there seemed 
to be no depth due to a "restless" appearance and percepts resembling those in binocular rivalry. This subject reported that he had used a more lenient criterion for this reason, namely the disappearance of all cues pointing to the hidden square. The low stereoscopic limits of $16-19$ min were obtained for two subjects in special experimental runs in which they had tried fixing their gaze as rigidly as possible on the fixation square. Such rigid fixation of gaze is known to interfere with the perception of stereoscopic depth (Linschoten, 1956; Ogle, 1952), so it is not surprising that the stereoscopic limits are lower under these conditions.

The typical values of $29-46 \mathrm{~min}$ were obtained while the subjects were looking "globally" at the RD stereogram by primarily paying attention to the stereoscopic depth in the hidden square and not looking consciously at the individual elements of the RD pattern. When the subjects who did not tend to the above-mentioned suppression effect switched their attention to these individual elements, in particular to the easily recognizable fixation square, above the diplopia threshold, this square was perceived as being double. At or just below the stereoscopic limits, this could only be done for a short time, because continued conscious local observation made the eyes gradually return from their divergent position (of 4-11 deg) to a more parallel setting so that stereopsis could not be regained.

A noteworthy finding is that the stereoscopic limits for the two subjects with suppression are comparable with those in subjects with normal binocular single vision. This again suggests that stereoscopic depth and what precisely is seen in depth may be processed fairly independently of one another in the central nervous system.

The above-mentioned "global" observation was studied in greater detail in one of the subjects because this subject (R.R.) reported that he could maintain motor fusion only when he violated the instruction that he should look at the upper righthand corner of the hidden square during the determination of the stereoscopic limits. Where he did look can be approximately inferred from Figure 6A, which gives the coordinates of afterimages imprinted during global observation with reference to afterimages imprinted during "local" observation of the fixation square. The fixation square and the hidden square are drawn to scale at the appropriate positions. Since the coordinates are plotted (with inverted polarity) relative to the center of the fixation square, the various points in the figure represent approximate successive directions of gaze within the stereogram. Figure 6B shows the coordinates of afterimages imprinted when the subject is directing his gaze to a certain prescribed element in the $\mathrm{RD}$ pattern (with $\mathrm{x}, \mathrm{y}$ coordinates of $-17.5,-36 \mathrm{~min}$ ).

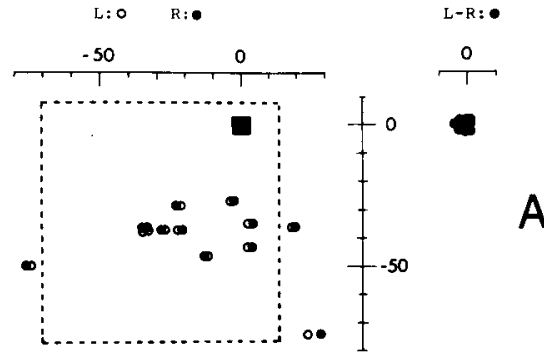

A

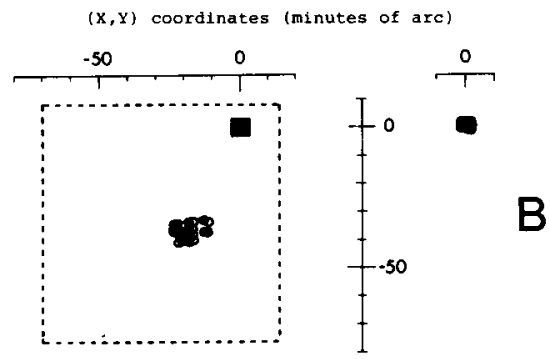

$(X, Y)$ coordinates (minutes of arc)

Figure 6. (A) $x, y$ coordinates (see text) of afterimages imprinted during "global" observation (see text), relative to the afterimages imprinted during fixation on the fixation square. (B) $x, y$ coordinates of afterimages imprinted during fixation on a prescribed element of the RD pattern (at $x, y=-17.5 \mathrm{~min}$, $36 \mathrm{~min}$ ), relative to the afterimages imprinted during fixation on the fixation square. In the left-hand part of $A$ and $B$, coordinates are given for the left eye (open circles) and the right eye (solid circles). In the right-hand part of $A$ and $B$, the discrepancies between these coordinates are plotted (solid circles). The positions of the fixation square (black square at 0,0 ) and the hidden square (large square drawn with broken line) are also shown in $A$ and $B$.

Comparison of Figures $6 \mathrm{~A}$ and $6 \mathrm{~B}$ suggests that one of the characteristics of global observation in the "no-pulling" condition is that the direction of gaze is extremely inaccurate and restricted only by the borders of the hidden square, while ocular alignment is as accurate as it is for local observation.

\section{Compatibility of Stereoscopic Limits for RD Stereograms and the Precision of Stereoscopic Depth Perception from Double Images of Elementary Line Stimuli}

The percepts observed at the stereoscopic limit for the RD stereogram were similar to those described by Ogle $(1952,1953)$ at the limit of patent stereopsis for elementary line stimuli in which depth is perceived from double images. This suggests that we are dealing with the same perceptual phenomenon. If so, there should also be a quantitative agreement between the stereoscopic limits for the RD stereogram and "the" precision of stereoscopic depth from diplopic images of elementary line stimuli in the fovea. To our knowledge, only Blakemore (1970), Ogle (1953), and Reading (1970) have presented data that can be used for a prediction of the 
stereoscopic limits. We wish to stress that these data were obtained under stimulus condition that differed from those used in the present study. Consequently, conclusions drawn from using these data for a prediction of stereoscopic limits for the RD stereogram can be only suggestive. The reader should, however, bear in mind that Fender and Julesz (1967, p. 820) also used Ogle's data base with regard to the fusion of elementary line stimuli in order to arrive at their conclusion that there were special neuronal processes that might raise the fusional limits for horizontal disparity in RD stereograms above those for elementary line stereograms.

Blakemore (1970), Ogle (1953), and Reading (1970) determined the frequency of seeing a test line in front of a reference line in a two-alternative forced choice procedure, when seeing the test line behind the reference line was the second alternative, as a function of the disparity difference between the two laterally separated (Ogle, 1953; Reading, 1970 ) or vertically separated (Blakemore, 1970) vertical lines with the mean horizontal disparity as parameter. These data were used to construct psychometric curves, the underlying standard deviation (SD) (Ogle, 1953; Reading, 1970) or the 0.677 SD value (Blakemore, 1970) of which was specified. A 1-SD criterion and a 0.677-SD criterion correspond to a large amount $(16 \%$ and $25 \%$, respectively, in the case of a Gaussian distribution) of false-positive classifications. Subjects tend to use more lenient criteria in a classification procedure corresponding to a much smaller amount of falsepositive classifications (typically much smaller than $5 \%$ ). In fact, the "threshold" in a classification procedure was found to be a factor of 2-3 larger than this SD (Duwaer \& Van den Brink, 1981a, 1981b); we took the value of $2.5 \mathrm{SD}$ for our further considerations. It is noteworthy that this choice is not very critical, since the value of SD increases rapidly with increasing mean horizontal disparity in the relevant range. When this threshold is to determine the disappearance of relative depth in the hidden square of the RD stereogram, it should be equal to $8 \mathrm{~min}$, which is the relative disparity in the hidden square. Figure 7 shows the disparities at which 2.5 SD equals $8 \mathrm{~min}$, according to the data of Blakemore, Ogle, and Reading. The expected range on the basis of Blakemore's (1970, Figures 5 and 6) data is indicated by the shaded area marked with "1." These data were obtained with $0.1-\mathrm{sec}$ presentations of two vertical lines above each other at 0 deg eccentricity. The expected range on the basis of Ogle's (1953, Figure 3) data is indicated by the shaded area marked with " 2 ." These data were obtained with 0.2-sec presentations (in which most spatial thresholds reach their asymptotic value for continuous presentation) of the two vertical lines on

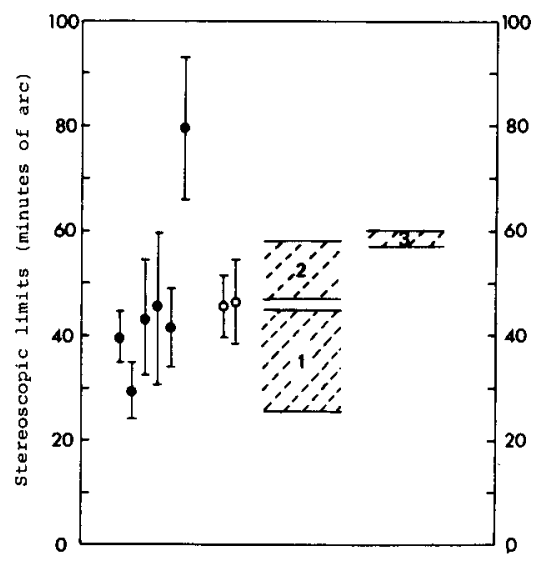

Figure 7. Comparison of stereoscopic limits obtained in the present study (circles with vertical bars of length 2 SDs, see Figure $5 \mathrm{C}$ ) and the expected range of the stereoscopic limits (shaded areas) on the basis of Blakemore's data (1970, "1"), Ogle's data (1953, “2") and Reading's data (1970, “3") about the perception of relative depth between diplopic images (see text).

opposite sides of the fixation point at 1-deg eccentricity (which is about equal to the mean eccentricity at which the relative disparity is determined in the RD stereogram). The shaded area marked with " 3 " indicates the expected range on the basis of Reading's (1970, Figure 3) data obtained with repeated 0.01 -sec presentations of the two vertical lines on opposite sides on the fixation point at 1.8deg eccentricity.

Comparison of the expected ranges for the stereoscopic limits and the stereoscopic limits actually measured (indicated in Figure 7 by the data points) shows that there does not seem to be a real discrepancy between the predicted and measured stereoscopic limits. Had there been special neuronal processes raising fusional limits for complex stimuli during slow disparity increase, as suggested by Fender and Julesz (1967), the fusional limits actually measured should have been much larger than the predicted ones (1) because of the much larger complexity of the RD stereogram, and (2) because horizontal disparity was slowly increased in the RD stereogram and not in the line stereograms used by Blakemore (1970), Ogle (1953), and Reading (1970).

The lack of discrepancy between the measured stereoscopic limits and those predicted on the basis of data available in the literature on the precision of stereoscopic depth from diplopic images of elementary stimuli thus suggests once more that there are no essential differences in the neuronal processing of horizontal retinal disparity in RD stereograms and in elementary line stereograms: Both types of stereograms seem to be capable of initiating neuronal processes that can bridge horizontal retinal disparities of up to at least $1 \mathrm{deg}$, leading to stereoscopic percepts (but not to singleness 
of binocular vision). The above-mentioned lack of discrepancy also supports the hypothesis that the stereoscopic limits for RD stereograms obtained with slow disparity increase are determined by the limited precision of stereoscopic depth perception from diplopic local images.

\section{Comparison with the Fusional Limits}

\section{Reported by Fender and Julesz (1967) and}

\section{Tyler and Julesz (1980)}

Even the stereoscopic limits we obtained during the slow temporalward pulling of the RD patterns were much smaller (by a factor of 1.5-4) than the fusional limits reported by Fender and Julesz (1967) for the same RD patterns with binocular stabilization. The reason for this discrepancy is not immediately apparent. The results obtained by Diner (1978) suggest that this discrepancy is not necessarily due to the stabilization of the stimulus on the retinas. Diner repeated Fender and Julesz's determination of fusional limits for binocularly stabilized bars and, like us, found values that were much smaller (by a factor of 3) than those of Fender and Julesz. Contrary to Fender and Julesz (1967), Diner (1978), by applying subatmospheric pressure, made sure that the contact lenses used for the image stabilization did not slip across the corneas, and Diner (1978) paid much attention to the perceptual criterion for singleness of binocular vision (see Introduction).

An interesting finding is that our stereoscopic limits are comparable to the values of $35-43 \mathrm{~min}$ reported by Tyler and Julesz (1980) for the relative disparity between surround and hidden figurewith the same area as the hidden figure used in the present study-at which stereoscopic depth disappears. However, the latter values were obtained with presentation times of $\mathbf{4 8} \mathrm{msec}$, which is shorter than the $0.2 \mathrm{sec}$ at which most spatial thresholds reach their asymptotic value for continuous presentation (which is what was used in the present study). Lehmkuhle and Fox (1980) found an improved depth perception in RD stereograms for higher presentation times-in the range of 68 to $160 \mathrm{msec}$. Such an improved depth perception is likely to be associated with a larger disparity limit. This suggests that Tyler and Julesz (1980) would have found larger disparity limits had they used a longer presentation time. Their disparity limits would then have been larger than the stereoscopic limits found in the present study, which is to be expected given the fact that Tyler and Julesz (1980) determined the disparity limits of stereopsis with a less demanding perceptual criterion (see Introduction). ${ }^{2}$

\section{Hysteresis in the Fusional Limits for RD Stereograms}

Fender and Julesz (1967) reported a new hysteresis phenomenon in stereopsis which was particularly impressive in the RD stereograms: the disparity limits for the breakaway of fusion are much larger (by a factor of up to 20) than the disparities at which refusion occurs. Fender and Julesz (1967) ascribed this hysteresis to the occurrence of special neuronal processes that raise the diplopia thresholds once fusion is established. However, the good agreement between the stereoscopic limits for an RD stereogram found in the present study and the limits of patent stereopsis for elementary line stimuli suggests quite a different interpretation of the hysteresis phenomenon, namely that the presence of so many dots in an RD stereogram hinders the spontaneous development of patent stereopsis from double images that may be observed in elementary line stereograms, as it becomes much more difficult for the visual system to establish which elements in the stereogram correspond to each other. As a result, the neuronal processes underlying patent stereopsis are not initiated, or are initiated much less adequately, so that the fusional limits are lowered. When the visual system is allowed to solve this correspondence problem in the much less demanding situation of near-zero overall disparity, patent stereopsis can become operative so that the fusional limits are no longer artificially lowered.

It is noteworthy that there are indications in the literature that patent stereopsis can become operative in RD stereograms not only by slowly increasing the disparity, but also by flashing the stereogram with a fixed disparity (Lehmkuhle \& Fox, 1980; Tyler \& Julesz, 1980). This extends Ogle's finding of superior stereoscopic processing of tachistoscopically presented line stereograms (Ogle, 1970).

\section{Accuracy of Horizontal Ocular Alignment on the Unpulled RD Stereogram}

Our results also throw light on the important and closely related question of the accuracy of horizontal ocular alignment on an RD stereogram under natural viewing conditions, when the disparity is well within the limits of motor fusion. This accuracy determines the range of horizontal fixation disparities that is being tolerated sensorially when the subject perceives a single fused RD pattern. Fender and Julesz (1967) claim that the ocular alignment is comparatively inaccurate ( $\mathrm{SD}=7-8 \mathrm{~min}$ ), which supports their finding of comparatively high limits for the breakaway of fusion.

We estimated the accuracy of horizontal ocular alignment on the RD stereogram from the spread of the induced horizontal disparity observed without temporalward pulling. This spread is determined by two independent samples of the distribution of ocular alignment (with variance $\sigma^{2}$ oa -obtained with two successively imprinted afterimages in each eye-and a sample of the distribution of the accuracy of the psychophysical assessment of the coordinates (with variance $\sigma_{c e}^{2}$ ). The variance of the 
distribution of induced horizontal disparities $\boldsymbol{\sigma}^{2}$ id may, therefore, be written $\sigma_{\text {id }}^{2}=2 \sigma^{2}{ }^{2}+\sigma_{c e}^{2}$. So, $\sigma_{\mathrm{oa}}=\sqrt{\sigma^{2}{ }_{\text {id }}-\sigma^{2}} / \sqrt{2}<\sigma_{\mathrm{id}} / \sqrt{2}$. The upper limits of the standard deviation $\sigma_{\mathrm{oa}}$ of the ocular alignment for our test subjects are presented in Figure 8. It may be seen that we found these upper limits to vary between 0.7 and $2.1 \mathrm{~min}$ in subjects with normal binocular single vision. These values agree with those reported earlier for elementary line stimuli (Duwaer, 1982a, 1982b; Duwaer \& Van den Brink, 1981b; Riggs \& Niehl, 1960), indicating that the diplopia thresholds for RD patterns need not be larger than those for elementary line stimuli.

The ocular alignment was found to be remarkably inaccurate in one of our subjects, who had a tendency to suppression of the image in one eye. This is not surprising, since ocular alignment does not need to be accurate because of the absence of image doubling up to fixation disparities of $40 \mathrm{~min}$. Nevertheless, the other subject manifesting this suppression effect did have accurate ocular alignment. This may indicate that the suppression effect does not necessarily occur at the input of the vergence system controlling the horizontal fusional eye movements.

\section{Mean Horizontal Fixation Disparity \\ During Fusion of Unpulled RD Stereograms}

It should be noted that the above-mentioned statements about the accuracy of ocular alignment concern the spread of ocular alignment around a mean value and not the mean value itself. The occurrence of a systematic error in this mean value, that is, of

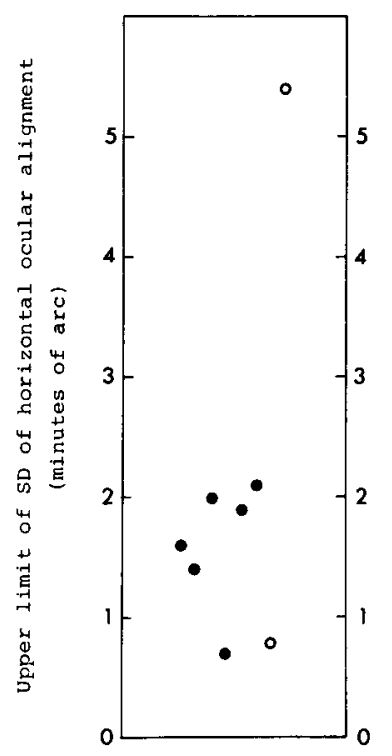

Figure 8. Upper limits of the SD of the distribution of ocular alignment in the horizontal direction for subjects manifesting a suppression effect (open circles) and for subjects without such a suppression effect (solid circles). a mean fixation disparity, was studied, using the afterimage method, in three subjects, A.L.D., R.R., and C.J.K., the last mentioned of whom has eccentric right-eye fixation. To this end, we imprinted an afterimage in the left eye during monocular observation of an RD element $\mathbf{1 3 . 2}$ min below the center of the fixation square with this eye, another afterimage in the right eye during monocular observation of the same RD element with the right eye, and a second afterimage in both eyes during binocular observation of the center of the fixation square; we then asked the subject to estimate the coordinates of the afterimage imprinted during monocular observation with reference to that imprinted during binocular observation for each eye separately. The coordinates thus obtained are plotted with open circles in Figure 9 for the three subjects tested. The coordinates of the RD element which is observed monocularly is indicated by ".+ "The mean values of the coordinates (represented by asterisks) suggest (Hebbard, 1962) that Subject A.L.D. looks behind the fixation square during binocular observation, with an equivalent underconvergence of $-4.8 \mathrm{~min}$. This agrees well with the horizontal fixation disparity of $\mathbf{- 5 . 2}$ min estimated with dichoptic nonius lines. The coordinates obtained from Subject R.R. similarly suggest that this subject looks in front of the fixation square during binocular observation with an equivalent overconvergence of $+2.6 \mathrm{~min}$, which agrees with the horizontal fixation disparity of $+2.3 \mathrm{~min}$ measured with dichoptic nonius lines.

A totally different situation was found in Subject C.J.K., who has eccentric right-eye fixation. The measured coordinates indicate a horizontal disparity of $-13.4 \mathrm{~min}$ between binocular and monocular fixation here, while the dichoptic nonius lines indicate a horizontal fixation disparity of $-0.8 \mathrm{~min}$. This subject has apparently developed anomalous binocular correspondence in which the egocentric localization of the images in both eyes has been changed with respect to the monocular egocentric localizations (cf. Crone, 1973). It is noteworthy that the correspondence anomaly of Subject C.J.K. has not affected the accuracy of ocular alignment (SD $<1.4 \mathrm{~min}$ ) or the limit of divergent motor fusion (11 deg).

Since the mean fixation disparity estimated by the afterimage method can apparently not be interpreted in the same way for all subjects, the data on fusional limits presented above have not been corrected for nonzero mean fixation disparity at the initial separation.

\section{Rigidity of Binocular Correspondence During Fusion of Temporalward Pulled RD Stereogram}

According to contemporary theories about stereopsis (e.g., Nelson, 1975, 1977), binocular cor- 

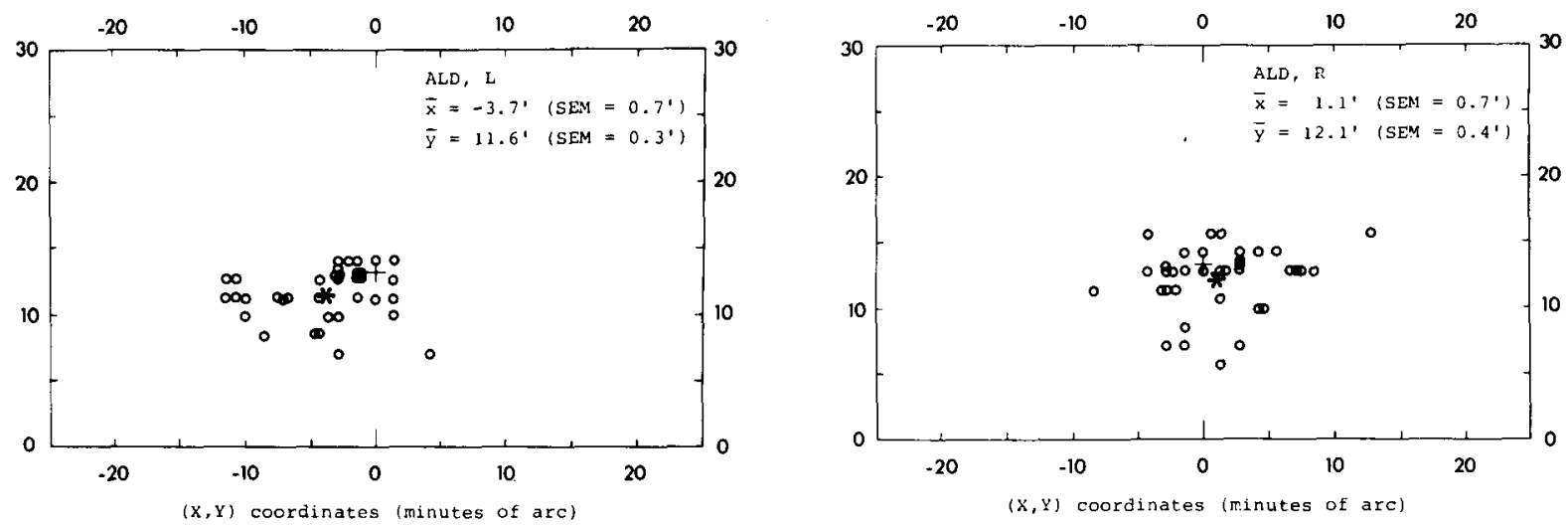

A
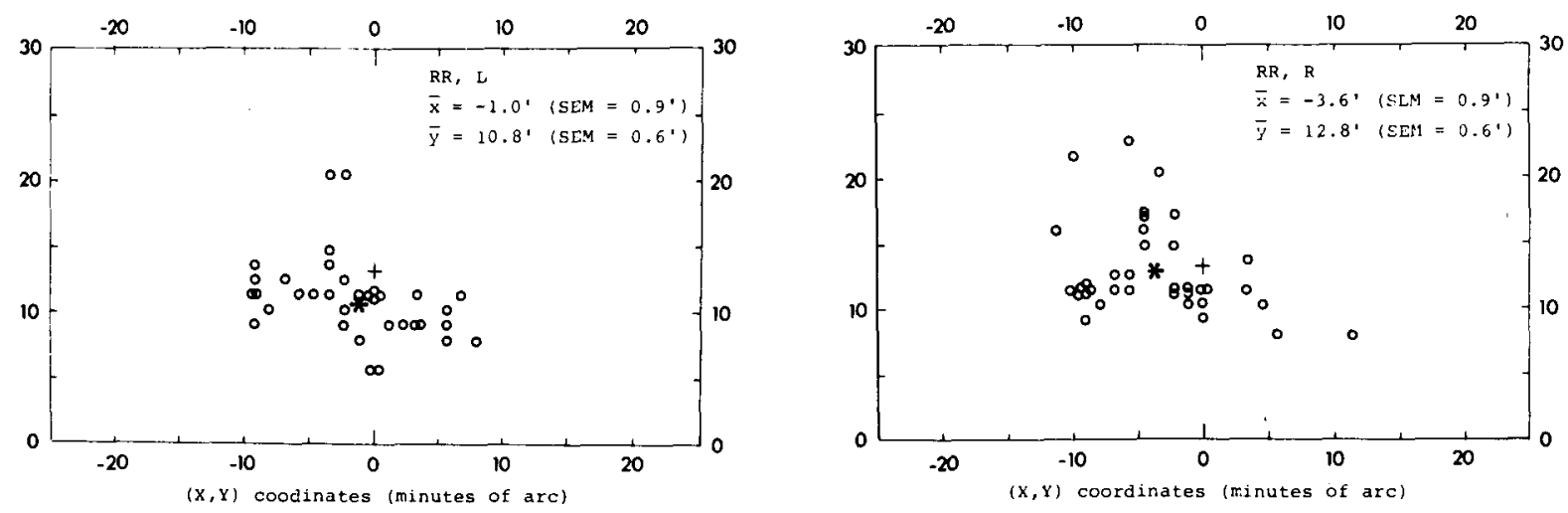

B
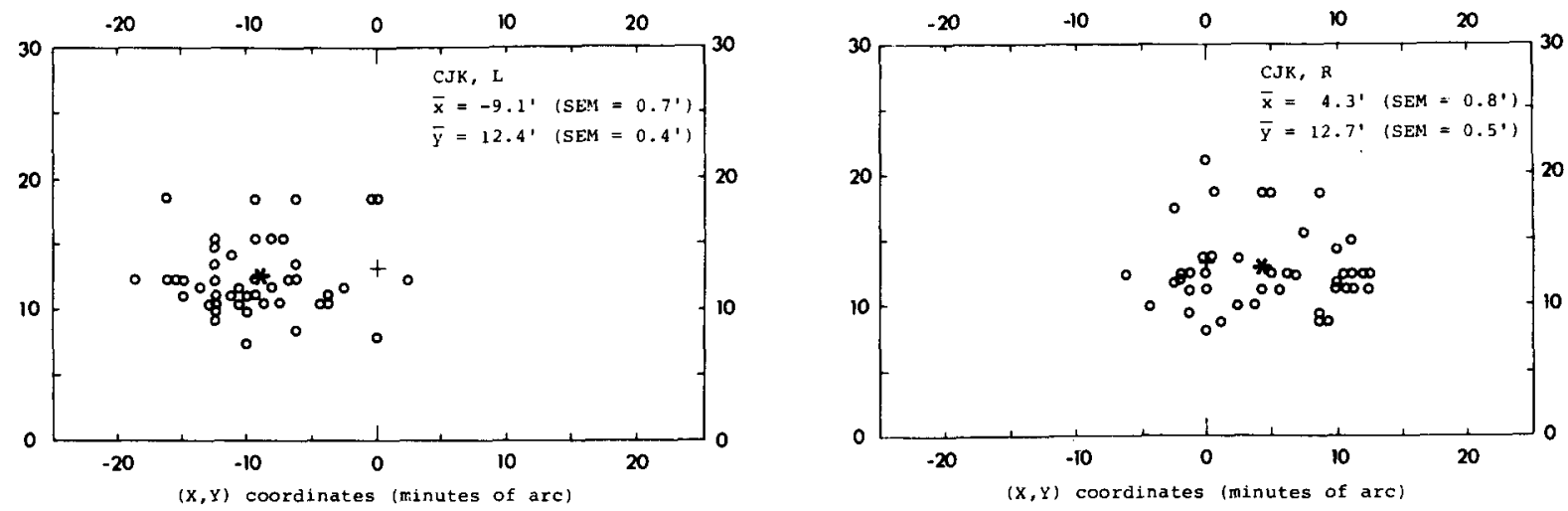

Figure 9. $x, y$ coordinates (open circles) of afterimages imprinted during monocular fixation of an RD element, the physical coordinates of which are represented by " + ," relative to afterimages imprinted during binocular fixation of the fixation square. The asterisk represents the mean values of the coordinates. (A) is for Subject A.L.D., (B) is for Subject R.R., and (C) is for Subject C.J.D. (who has eccentric right-eye fixation).

respondence should change under the influence of fusional stress induced by the fusion occurring (as evidenced by the perception of stereoscopic depth) when the RD pattern is pulled temporalward. The latter was studied in three subjects in an experiment with vertical dichoptic nonius lines (made of 3-minwide black tape stuck on the patterns) positioned one above the other, with the line in the right-eye 
pattern displaced farther to the left with respect to the fixation square than the line in the left-eye pattern, resulting in a horizontal disparity between the nonius lines. The subject slowly increased the separation between the RD patterns until the dichoptic nonius lines-the perceived relative position of which varied continuously-could be seen in line. The subject tried to actuate the flashes at the moment of alignment-which caused afterimages to be imprinted on the retinas of both eyes-and then estimated the relative horizontal displacement of the centers of the two imprinted afterimages while looking at the TV screen with both eyes open. This binocular viewing condition involves very little fusional stress, certainly much less than in the previous condition with temporalward-pulled RD patterns. In spite of this, the horizontal disparity between the afterimages thus observed did not differ from the horizontal disparity between the nonius lines, which corresponded to the observed horizontal disparity under conditions of heavy fusional stress. It amounted to $24 \mathrm{~min}$ (SD $=2 \mathrm{~min}$ ) for nonius lines with 25 -min horizontal disparity in Subject A.L.D., $23 \mathrm{~min}(\mathrm{SD}=2.5 \mathrm{~min}$ ) for nonius lines with $23-\mathrm{min}$ horizontal disparity in Subject R.R., and $27 \mathrm{~min}$ $(\mathrm{SD}=3.5 \mathrm{~min})$ for nonius lines with $27-\mathrm{min}$ horizontal disparity in Subject C.J.K. (who has anomalous binocular correspondence). This finding of rigid binocular correspondence agrees with similar findings by Flom and Eskridge (1968), who showed that the binocular correspondence in subjects with normal binocular vision remains fixed to within the visual resolving power when the viewing distance changes between $10 \mathrm{~cm}$ and $6 \mathrm{~m}$. We would like to stress that the finding of rigid binocular correspondence defies the predictions of contemporary theories about stereopsis (e.g., Nelson, 1975, 1977).

\section{Conclusions}

The diplopia thresholds for an RD stereogram were found to be consistently smaller than the classical upper limit (of about $0.3 \mathrm{deg}$ ) reported for elementary line stimuli.

The stereoscopic limits for an RD stereogram were found to be in the range 0.5-1.3 deg, which is compatible with the limits reported for patent stereopsis from diplopic images in elementary line stereograms.

The accuracy of ocular alignment on an RD stereogram (if the two eyes are parallel) was found to be comparable to previously reported data on the accuracy of ocular alignment on elementary line stimuli.

The present results defy various predictions of several contemporary theories about stereopsis (models of Julesz, 1971, and Nelson, 1975, 1977).

The overall results suggest that there are no spe- cial neuronal processes that may raise the fusional limits for RD stereograms above those for elementary line stereograms. Previous claims that such special neuronal processes had been found seem to be due to the use of different criteria for fusion for RD stereograms and for elementary line stereograms.

The large hysteresis effect that has been observed for the fusional limits of RD stereograms should be ascribed not to a raising of the classical diplopia threshold due to special neuronal processes initiated by RD stereograms, but to a lowering of the classical limits of patent stereopsis from double images due to the increased difficulty of solving the correspondence problem in RD stereograms.

The results provide evidence for the hypothesis that the neuronal pathways underlying stereopsis and singleness of binocular vision bifurcate into two fairly independent branches.

\section{REFERENCES}

Blakemore, C. The range and scope of binocular depth discrimination in man. Journal of Physiology (London), 1970, 211, 599-622.

Crone, R. A. Diplopia. New York: Elsevier, 1973.

Crone, R. A., \& Hardjowijoto, $\mathbf{S}$. What is normal binocular vision? Documenta Ophthalmologica, 1979, 47, 163-199.

DINER, D. B. Hysteresis in human binocular fusion: $A$ second look. Doctoral dissertation, California Institute of Technology, 1978.

DitchBuRn, R. W. Eye-movements and visual perception. Oxford: Clarendon Press, 1973.

DRYSDALE, A. E. The visibility of retinal blood vessels. Vision Research, 1975, 15, 813-818.

Duwaer, A. L., \& VAN DEN Brink, G. What is the diplopia threshold? Perception \& Psychophysics, 1981, 29, 295-309. (a)

Duwaer, A. L., \& Van den Brink, G. Foveal diplopia thresholds and fixation disparities. Perception \& Psychophysics, 1981, 30, 321-329. (b)

Duwaer, A. L. Assessment of retinal image displacement during active head movement using an afterimage method. Vision Research, 1982, 22, 1379-1388. (a)

DuwAER, A. L. Nonmotor component of fusional response to vertical disparity: A second look using an afterimage method. Journal of the Optical Society of America, 1982, 72, 871-877. (b)

Fender, D., \& Julesz, B. Extension of Panum's fusional area in binocularly stabilized vision. Journal of the Optical Society of America, 1967, 57, 819-830.

Flom, M. C., \& Eskridge, J. B. Change in retinal correspondence with viewing distance. Journal of the American Optometric Association, 1968, 39, 1094-1097.

HebraRd, F. W. Comparison of subjective and objective measurements of fixation disparity. Journal of the Optical Society of America, 1962, 52, 706-712.

Julesz, B. Foundations of cyclopean perception. Chicago: University of Chicago Press, 1971.

Lehm KuHLE, S., \& Fox, R. Effect of depth separation on metacontrast masking. Journal of Experimental Psychology: Human Perception and Performance, 1980, 6, 605-621.

Linschote N, J. Structuranalyse der binokularen tiefenwahrnehmung. Thesis, University of Utrecht, 1956.

Magnussen, S., \& Tuorjussen, T. Sustained visual afterimages. Vision Research, 1974, 14, 743-744.

Nelson, J. I. Globality and stereoscopic fusion in binocular vision. Journal of Theoretical Biology, 1975, 49, 1-88. 
NELson, J. I. The plasticity of correspondence: Aftereffects, illusions and horopter shifts in depth perception. Journal of Theoretical Biology, 1977, 66, 203-206.

Ogle, K. N. Disparity limits of stereopsis. Archives of Ophthalmology, 1952, 48, 50-60.

Ogle, K. N. Precision and validity of stereoscopic depth perception from double images. Journal of the Optical Society of America, 1953, 43, 906-913.

Oale, K. N. Stereopsis and vertical disparity. Archives of Ophthalmology, 1955, 53, 495-504.

OGLE, K. N. Researches in binocular vision. New York: Hafner, 1970.

Ogle, K. N., \& Wakefield, J. M. Stereoscopic depth and binocular rivalry. Vision Research, 1967, 7, 89-98.

Reading, $R$. W. The threshold of distance discrimination for objects located outside Panum's area. American Journal of Optometry, 1970, 47, 99-105.

RICH ARDs, W. Stereopsis with and without monocular contours. Vision Research, 1977, 17, 967-969.

RIcGs, L. A., \& NiEhL, E. W. Eye movements recorded during convergence and divergence. Journal of the Optical Society of America, 1960, 50, 913-920.

Tyler, C. W., \& Julesz, B. On the depth of the cyclopean retina. Experimental Brain Research, 1980, 40, 196-202.

Westheimer, G., \& Tanzman, I. J. Qualitative depth localization with diplopic images. Journal of the Optical Society of America, 1956, 46, 116-117.

\section{NOTE}

1. The foveal diplopia thresholds for 30 -min vertical lines (width $1.2 \mathrm{~min}$ ) for which the horizontal disparity slowly increases (with a rate of $0.4 \mathrm{~min} / \mathrm{sec}$ ) were found to amount to 3.8-7.9 $\mathrm{min}$ for Subject A.L.D. and 5.8-16.4 $\mathrm{min}$ for Subject C.J.D., depending on the criterion used for diplopia.

The corresponding thresholds for slow disparity decrease were 3.9-7.5 min for Subject A.L.D. and 6.3-13.8 min for Subject C.J.D. Previously published data were mean values of the thresholds obtained with slow disparity increase and slow disparity decrease (cf. Duwaer \& Van den Brink, 1981a).

2. Tyler and Julesz (1980) did find higher disparity limits for RD stereograms when they used a hidden figure with a larger area. Larger areas result in higher eccentricities of stimulation, for which the fusional limits are known to be larger (Ogle, 1952) and for which stereoscopic depth discrimination at large disparities is better (Blakemore, 1970; Ogle, 1953). Consequently, the disparity has to be increased further in order to remove all subthreshold stimulation (cf. Tyler \& Julesz, 1980, p. 198). All comparisons between fusional limits in the present study are based on data obtained with similar maximum eccentricities of stimulation and similar stimulus dimensions.

(Manuscript received April 23, 1982;

revision accepted for publication December 22, 1982.) 